\title{
Características demográficas e audiológicas da população pediátrica de um centro de referência em saúde auditiva de São Paulo
}

\author{
Demographic and audiological characteristics \\ of a pediatric population in a hearing health \\ care center in São Paulo
}

\section{Características demográficas y audiológicas de la población pediátrica de un centro de referencia en salud auditiva de Sao Paulo}

\author{
Silvia Napole Fichino* \\ Vera Lucia Ferreira Avelino* \\ Doris Ruthy Lewis*
}

\section{Resumo}

Introdução: A criação de políticas públicas regulamentando ações na atenção básica de promoção à saúde auditiva, prevenção e identificação de problemas auditivos, garante a reabilitação, dando proteção às pessoas com deficiência. Objetivo: Caracterizar os dados demográficos e audiológicos dos usuários de um centro de referência em saúde auditiva, do município de São Paulo. Método: Trata-se de estudo de caráter descritivo e retrospectivo, desenvolvido no Centro Audição na Criança (CeAC/DERDIC/ PUCSP). Foram analisados 857 prontuários de pacientes que compareceram para avaliação audiológica e concluíram o diagnóstico, entre agosto/2010 e junho/2015. Foram extraídos os seguintes dados dos prontuários: sexo, data de nascimento, zona de moradia, realização da triagem auditiva neonatal (TAN), indicadores de risco para deficiência auditiva na história da criança, origem do encaminhamento, idade na primeira consulta, idade na finalização do diagnóstico audiológico e resultado do mesmo. Resultados: $64,2 \%$ das crianças atendidas residem na região Sul de São Paulo; 2 meses foi a idade média no início do

* Pontifícia Universidade Católica de São Paulo, São Paulo, SP, Brasil

Contribuição dos autores:

SNF - Contribuiu na elaboração da redação do artigo.

VLFA - Foi a pesquisadora do estudo coletando e analisando os dados.

DRL - Orientou a pesquisa e a elaboração do artigo.

E-mail para correspondência: Silvia Napole Fichino snfichino@gmail.com Recebido: $15 / 12 / 2017$

Aprovado: 25/07/2018 
diagnóstico; $54,8 \%$ das queixas foi suspeita de perdas auditivas seguida da necessidade de diagnóstico por falha na TAN $(29,6 \%) ; 57,8 \%$ das crianças realizaram a TAN; $50,2 \%$ finalizaram o diagnóstico com idade superior a um ano, seguida pelas crianças de 0 à 3 meses $(18,4 \%)$; o diagnóstico encontrado nas crianças foi de $22,4 \%$ de perdas auditivas sensórioneurais bilateralmente, $17,7 \%$ de perdas condutivas. Conclusão: A maioria da população atendida se origina em queixas de perdas auditivas e não pela demanda proveniente das maternidades que realizam a TAN. Nota-se ainda que o referido centro atende a população de sua área de referência.

Palavras-chave: Perda auditiva; Criança; Políticas públicas; Diagnóstico precoce; Programas de rastreamento.

\section{Abstract}

Introduction: The creation of public policies regulating actions in primary care to promote hearing health care, prevention and identification of hearing loss, ensures rehabilitation, providing support and protection of persons with disabilities, in addition to health. Objective: To characterize demographic and audiological data of users of a hearing health care center, in São Paulo City. Method: This is a descriptive and retrospective observational study developed at the Hearing in Children Center (CeAC). There were analyzed 857 patients' recordings of those who concluded the audiological assessment, from August 2010 to June 2015. The following data were extracted from the recordings: gender, date of birth, dwelling area, newborn hearing screening (NHS) tests performed, risk indicators for hearing loss in the children's history, origin of the referral, age at the first visit, age at diagnosis, and results for hearing status. Results: $64.2 \%$ of the children live in the South region of São Paulo city; 2 months old was the mean age at the beginning of the diagnosis; $54,8 \%$ of the complaints were of hearing loss followed by refers after NHS (29.6\%); $57.8 \%$ of the children performed NHS; $50,2 \%$ of children completed the diagnosis in the age group over one year old; auditory status was found to be $22,4 \%$ for bilaterally sensorial hearing loss, $17,7 \%$ for conductive hearing loss. Conclusion: The principal reason for searching diagnosis was hearing loss, and not because of refers after NHS. The center assists specially the children who live in the area for which the center is responsible, according to the hearing health policies.

Keywords: Hearing loss; Child; Public policy; Early diagnosis; Tracking programs.

\section{Resumen}

Introducción: La creación de políticas públicas regulando acciones en la atención básica de promoción a la salud auditiva, prevención e identificación de problemas auditivos, garantiza la rehabilitación, dando amparo a las personas con discapacidad. Objetivo: Caracterizar los datos demográficos y audiológicos de los usuarios de un centro de referencia en salud auditiva. Método: Se trata de un estudio de carácter descriptivo y retrospectivo, desarrollado en el Centro AudicióndelNiño (CeAC/DERDIC/PUCSP). Se analizaron 857 prontuarios de pacientes que comparecieron para evaluación audiológica y concluyeron el diagnóstico, entre agosto/2010 a junio/2015. Se tomaron los siguientes datos: sexo, fecha de nacimiento, zona de vivienda, realización de lo rastreo auditivo neonatal(TAN), indicadores de riesgo para deficiencia auditivaen la historia del niño, origen del encaminamiento, edad en la primera consulta, edad en la finalización del diagnóstico audiológico y su resultado. Resultados: $64,2 \%$ de los niños atendidos residen en la región Sur de São Paulo; 2 meses fue el promedio de edad al inicio del diagnóstico; 54,8\% de las quejas fue sospecha de pérdida auditiva, seguida de la necesidad de diagnóstico por fallo en el rastreo (29,6\%); 57,8\% de los niños realizaron rastreo; 50,2\% finalizó el diagnóstico con edad superior a un año, seguido por niños de 0 a 3 meses 18,4\%; el diagnostico encontrado fue de $22,4 \%$ de pérdidas auditivas sensorioneuronales bilaterales, $17,7 \%$ de pérdidas conductivas. Conclusión: La mayoría de la población atendida se origina a partir de quejas de pérdidas auditivas y no por demanda proveniente de las maternidades que realizan el rastreo. Se observa además que el referido centro atiende a la población de su área de referencia.

Palabras claves: Pérdida de la audición; Niño; Políticas públicas; Diagnóstico precoz; programas de rastreo. 


\section{Introdução}

A integridade do sistema auditivo é fundamental para o desenvolvimento da criança, tendo em vista que a audição é o meio para aquisição de habilidades de linguagem e fala, que fazem com que a criança possa organizar e entender o universo, transmitir informações, compreender o outro, interagir com o mundo e aprender ${ }^{1}$. Crianças que apresentam alterações auditivas podem ter o seu desenvolvimento de linguagem, fala, aprendizado, social e emocional afetados ${ }^{2}$. Desta forma, medidas devem ser tomadas para a identificação de alterações auditivas o mais cedo possível, visando o diagnóstico rápido e preciso, e uma intervenção terapêutica com a criança e com a família, minimizando os efeitos desta privação sensorial e aproveitando a plasticidade cerebral ${ }^{2,3}$.

Para que o diagnóstico precoce possa ocorrer, é importante que existam políticas públicas de saúde, em especial, aquelas voltadas à saúde auditiva, que garantam o direito à identificação, diagnóstico, intervenção e (re)habilitação da criança com deficiência auditiva, seguindo diretrizes para a organização e implantação de Redes Regionalizadas de Atenção à Saúde Auditiva ${ }^{4}$.

Em nosso país, existem diretrizes definidas na Portaria MS/GM n ${ }^{\circ} 835$, de 25 de abril de $2012^{5}$ e na Portaria MS/GM n ${ }^{\circ} 1.278$, de 20 de outubro de $1999^{6}$ que preconizam ações desde a identificação precoce, por meio da Triagem Auditiva Neonatal Universal (TANU), diagnóstico, e inclusive medidas de intervenção, por meio de adaptação de aparelho de amplificação sonora individual (AASI) e até mesmo o implante coclear (IC). Para todas as crianças com perdas auditivas que podem afetar o desenvolvimento da linguagem, ou consideradas incapacitantes, a terapia fonoaudiológica também é preconizada ${ }^{5,6}$.

Portanto, sendo a audição um fator considerado fundamental no processo de aquisição e desenvolvimento da linguagem e a detecção precoce da perda auditiva um fato de extrema importância no prognóstico da reabilitação, por minimizar os efeitos da deficiência auditiva sobre o indivíduo ${ }^{7}$ recomenda-se a realização da TANU até o primeiro mês de vida, e a conclusão do diagnóstico até o terceiro mês de vida ${ }^{8}$. Recomenda-se, ainda, iniciar a intervenção clínica imediatamente após o resultado positivo para deficiência auditiva, com uso de dispositivos eletrônicos, terapia fonoaudiológica, e orientação a pais e cuidadores ${ }^{7,8}$.

Em 2010, foi criado no Brasil o Comitê Multiprofissional em Saúde Auditiva (COMUSA), que referenda ações voltadas à saúde auditiva em todas as faixas etárias, com recomendações de boas práticas relacionadas à TANU, diagnóstico e intervenção precoce além de orientações sobre a necessidade de se aprimorar os processos envolvidos no programa de saúde auditiva9

Em 02 de Agosto de 2010, foi sancionada a Lei ${ }^{\circ} 12303$. No texto o autor apresenta a triagem auditiva neonatal (TAN) e resolve sobre a obrigatoriedade do exame de emissões otoacústicas (EOA) a todos os recém-nascidos, em âmbito nacional ${ }^{10}$.

Em 2012, foram publicados os Instrutivos da Saúde Auditiva, referenciando diretrizes para tratamento, reabilitação e/ou habilitação de pessoas com deficiência auditiva, física, intelectual e visual. Também regulamenta normas gerais de funcionamento dos Centros Especializados em Reabilitação (CER), com instalações físicas, horários de funcionamento e recursos humanos e materiais. Dentro das diretrizes para as pessoas com deficiência auditiva, também encontra disposto no programa o serviço para reabilitação/habilitação auditiva, bem como os critérios para a Avaliação Diagnóstica, indicação de próteses auditivas, e terapia fonoaudiológica ${ }^{11}$.

Uma pesquisa realizada em Campinas em duas clínicas especializadas em surdez estudou 320 prontuários de pacientes diagnosticados com surdez pré-lingual e identificaram que mesmo com a aprovação de projetos de leis referendando a TAN, os programas de identificação precoce da perda auditiva não têm atingido toda a população ${ }^{12}$. Nesse trabalho, a idade média com que a criança chegou à primeira consulta foi de 3 anos e 6 meses, e a perda auditiva foi suspeitada pelos pais entre 1 a 9 meses.

Em São Paulo, um estudo analisou a implementação das ações em saúde auditiva pesquisando os principais documentos relacionados à saúde auditiva por meio do sistema de informação, fóruns regionais e entrevistas com profissionais de três centros de alta complexidade. Foi possível identificar dois grandes marcos: a implantação da Política Nacional de Atenção à Saúde Auditiva (PNASA) e o "Plano Viver sem Limite". Os dados também evidenciaram um crescimento na concessão de aparelhos de amplificação sonora individual e 
implantes cocleares, além da realização de procedimentos para identificação da perda auditiva. Porém, em relação ao acompanhamento e à terapia, os números ficaram abaixo do esperado. $\mathrm{O}$ estudo sugeriu um momento de mudanças, oportuno para discussão, requerendo esforços e diálogo entre todos envolvidos, para facilitar os processos e o acesso a uma vida mais saudável e justa, daqueles com alguma deficiência ${ }^{13}$.

Contudo, a PNASA possibilitou uma maior organização no atendimento à população com deficiência auditiva e aprimoramento das ações em saúde auditiva, uma vez que propõe uma rede hierarquizada, regionalizada e integrada, desde a atenção básica até a alta complexidade, tentando assim garantir a reabilitação necessária e o acesso aos serviços. Com isso, mais uma vez, a PNASA tem papel fundamental para a organização e acesso aos serviços de identificação, diagnóstico e reabilitação, facilitando o acesso aos serviços, estruturando uma rede de cuidados, de acordo com diferentes níveis de complexidade ${ }^{14}$.

Outro estudo, nacional, procurou avaliar a PNASA a partir da cobertura de serviços especializados e procedimentos diagnósticos em saúde auditiva de média e alta complexidade, por região e no Brasil. Foram utilizados os dados do Departamento de Informática do Sistema Único de Saúde (DATASUS), no período de 2004 a 2011. Identificou-se um aumento de $113 \%$ na cobertura de serviços e $61 \%$ na quantidade de procedimentos de diagnóstico em saúde auditiva de média e alta complexidade em todo o país. A região Norte do país apresentou $78 \%$ de aumento no número de procedimentos. Já a região Sudeste realiza proporcionalmente maior quantidade de procedimentos. Identificou-se, também, um aumento expressivo dos exames de emissões otoacústicas (EOA) para triagem auditiva, de estudos de EOA transientes e produto de distorção, como também dos exames de reavaliação diagnóstica de deficiência auditiva em pacientes maiores de três anos. Conclui-se que houve um aumento significativo de serviços e ações em atenção à saúde auditiva no Brasil desde a implantação da PNASA, mas, ainda persistem importantes desigualdades regionais na oferta de serviços ${ }^{15}$.

Embasado na PNASA e diretrizes nacionais e municipais, este artigo pretende caracterizar os dados demográficos e audiológicos dos usuários de um centro de referência em saúde auditiva do município de São Paulo.

\section{Método}

O estudo foi realizado no Centro Audição na Criança, clínica da Divisão de Educação e Reabilitação dos Distúrbios da Comunicação da Pontifícia Universidade Católica de São Paulo (CeAC-DERDIC/PUCSP), unidade credenciada da Rede de Atenção em Saúde Auditiva de São Paulo.

No primeiro momento, foi utilizado o Sistema de Agendamento para a busca dos pacientes agendados no CeAC no período de Agosto de 2010 a Junho de 2015. Este sistema trata-se de um programa geral da instituição utilizado para armazenar informações de agenda e cadastro de pacientes. Após este levantamento, foram identificados no arquivo da instituição 857 prontuários de pacientes que foram atendidos durante o período do estudo e com conclusão do diagnóstico audiológico e definição do status auditivo. Para auxiliar na coleta de dados, foi elaborado um formulário clínico com os dados necessários para a pesquisa, e que foram inseridos em uma planilha Excell para posterior análise estatística. De cada prontuário foram extraídas as seguintes informações: data de nascimento, idade no primeiro atendimento, região de moradia, origem e motivo do encaminhamento, presença de Indicador de risco para deficiência auditiva (IRDA), idade no término do diagnóstico, status auditivo após conclusão diagnóstica (resultado do diagnóstico). Os prontuários foram analisados por meio do registro da ficha de anamnese, folhas de evoluções médica e fonoaudiológica, e registro dos exames realizados para diagnóstico: Emissões Otoacústicas Evocadas (EOA), Potencial Evocado Auditivo de Tronco Encefálico (PEATE), audiometria de reforço visual (ARV) ou lúdica condicionada (ALC) e imitânciometria. Foram considerados indicadores de risco para Deficiência auditiva (IRDA) os indicados pelo Comitê Multiprofissional em Saúde Auditiva (COMUSA) e pelo Joint Committee on Infant Hearing $(\mathrm{JCIH})^{7,9}$.

Foi realizada análise descritiva dos dados, sendo elas: análise demográfica caracterizando gênero e idade da criança no primeiro atendimento, idade na conclusão do diagnóstico, procedência (cidade, Estado e região atual de moradia na cidade), origem do encaminhamento (serviços que realizaram o encaminhamento para o diagnóstico), se ocorreu a 
realização da TANU e onde foi realizada; caracterização audiológica caracterizando segundo presença de IRDA e resultado audiológico após avaliação realizada. Foi realizada análise inferencial a partir do cruzamento dos seguintes dados: realização da TANU segundo idade do primeiro atendimento; realização da TANU segundo idade na conclusão do diagnóstico; origem do encaminhamento segundo idade na conclusão do diagnóstico; resultado da TANU segundo resultado do diagnóstico.

\section{Resultados}

Foram encontrados 857 prontuários de pacientes que realizaram o diagnóstico audiológico no CeAC no período de agosto de 2010 a junho de 2015.

Assim, serão apresentados os seguintes resultados: distribuição de prontuários/pacientes pesquisados, idade no primeiro atendimento, região de moradia, motivo do encaminhamento para o diagnóstico audiológico, presença de IRDA, idade no diagnóstico e o status auditivo.

$\mathrm{O}$ resumo descritivo da idade em meses, no primeiro atendimento, é apresentado na Tabela 01. Podemos notar que, em todos os anos houve crianças em que o primeiro atendimento ocorreu antes da idade de 01 mês, contudo a maioria das crianças atendidas possuíam idade igual ou maior a 12 meses.

Tabela 1. Distribuições de frequências e porcentagens da faixa etária no primeiro atendimento por ano em que ocorreu o primeiro atendimento

\begin{tabular}{|c|c|c|c|c|c|}
\hline \multirow{2}{*}{ Ano $1^{\circ}$ atendimento } & \multicolumn{4}{|c|}{ Faixa etária no $1^{\circ}$ atendimento } & \multirow{2}{*}{ Total } \\
\hline & 0a3meses & 4a6meses & 7a12meses & mais que $12 \mathrm{~m}$ & \\
\hline \multirow[t]{2}{*}{2010} & 25 & 8 & 13 & 35 & 81 \\
\hline & $30,9 \%$ & $9,9 \%$ & $16,0 \%$ & $43,2 \%$ & $100,0 \%$ \\
\hline \multirow[t]{2}{*}{2011} & 33 & 15 & 25 & 68 & 141 \\
\hline & $23,4 \%$ & $10,6 \%$ & $17,7 \%$ & $48,2 \%$ & $100,0 \%$ \\
\hline \multirow[t]{2}{*}{2012} & 24 & 9 & 39 & 92 & 164 \\
\hline & $14,6 \%$ & $5,5 \%$ & $23,8 \%$ & $56,1 \%$ & $100,0 \%$ \\
\hline \multirow[t]{2}{*}{2013} & 47 & 30 & 25 & 84 & 186 \\
\hline & $25,3 \%$ & $16,1 \%$ & $13,4 \%$ & $45,2 \%$ & $100,0 \%$ \\
\hline \multirow[t]{2}{*}{2014} & 48 & 22 & 35 & 85 & 190 \\
\hline & $25,3 \%$ & $11,6 \%$ & $18,4 \%$ & $44,7 \%$ & $100,0 \%$ \\
\hline \multirow[t]{2}{*}{2015} & 21 & 8 & 21 & 45 & 95 \\
\hline & $22,1 \%$ & $8,4 \%$ & $22,1 \%$ & $47,4 \%$ & $100,0 \%$ \\
\hline \multirow[t]{2}{*}{ Total } & 198 & 92 & 158 & 409 & 857 \\
\hline & $23,1 \%$ & $10,7 \%$ & $18,4 \%$ & $47,7 \%$ & $100,0 \%$ \\
\hline
\end{tabular}

A maioria das crianças atendidas era moradora das Regiões Sul e Norte, e esse resultado deve-se ao fato de que a instituição é referência para tais regiões.

No que se refere à origem do encaminhamento, destaca-se que as Maternidades e os Centros Especializados em Reabilitação (CER) estão nos primeiros lugares como origem do encaminhamento. Com o início da TANU, no município, aumentou o número de encaminhamentos para diagnóstico após a falha na TANU, proveniente dos hospitais que a realizam.

A maioria das crianças realizou a TANU. Contudo, vale ressaltar que, as porcentagens de crianças que fizeram a TANU, a realizaram entre 2013 e 2015, observando-se maiores porcentagens quando comparadas com o período de 2010 a 2012. Mostra-se assim, uma evolução no número de crianças com o passar dos anos. 
Tabela 2. Distribuições de frequências e porcentagens da região de moradia por ano em que ocorreu o primeiro atendimento

\begin{tabular}{|c|c|c|c|c|c|c|c|}
\hline \multirow{2}{*}{ Região de moradia } & \multicolumn{6}{|c|}{ Ano $1^{\circ}$ atendimento } & \multirow{2}{*}{ Total } \\
\hline & 2010 & 2011 & 2012 & 2013 & 2014 & 2015 & \\
\hline \multirow[t]{2}{*}{ NORTE } & 16 & 29 & 24 & 49 & 45 & 22 & 185 \\
\hline & $19,8 \%$ & $20,6 \%$ & $14,6 \%$ & $26,3 \%$ & $23,7 \%$ & $23,2 \%$ & $21,6 \%$ \\
\hline \multirow[t]{2}{*}{ SUL } & 47 & 87 & 119 & 119 & 113 & 65 & 550 \\
\hline & $58,0 \%$ & $61,7 \%$ & $72,6 \%$ & $64,0 \%$ & $59,5 \%$ & $68,4 \%$ & $64,2 \%$ \\
\hline \multirow[t]{2}{*}{ LESTE } & 7 & 15 & 10 & 15 & 21 & 6 & 74 \\
\hline & $8,6 \%$ & $10,6 \%$ & $6,1 \%$ & $8,1 \%$ & $11,1 \%$ & $6,3 \%$ & $8,6 \%$ \\
\hline \multirow[t]{2}{*}{ OESTE } & 9 & 8 & 5 & 2 & 5 & 1 & 30 \\
\hline & $11,1 \%$ & $5,7 \%$ & $3,0 \%$ & $1,1 \%$ & $2,6 \%$ & $1,1 \%$ & $3,5 \%$ \\
\hline \multirow[t]{2}{*}{ CENTRAL } & 1 & 0 & 0 & 0 & 5 & 1 & 7 \\
\hline & $1,2 \%$ & $0,0 \%$ & $0,0 \%$ & $0,0 \%$ & $2,6 \%$ & $1,1 \%$ & $0,8 \%$ \\
\hline \multirow[t]{2}{*}{$\begin{array}{l}\text { METROPOLITANA/ } \\
\text { LITORAL }\end{array}$} & 1 & 2 & 6 & 1 & 1 & 0 & 10 \\
\hline & $1,2 \%$ & $1,4 \%$ & $3,6 \%$ & $0,5 \%$ & $0,5 \%$ & $0,0 \%$ & $1,2 \%$ \\
\hline \multirow[t]{2}{*}{ Total } & 81 & 141 & 164 & 186 & 190 & 95 & 857 \\
\hline & $100,0 \%$ & $100,0 \%$ & $100,0 \%$ & $100,0 \%$ & $100,0 \%$ & $100,0 \%$ & $100,0 \%$ \\
\hline
\end{tabular}

Tabela 3. Distribuições de frequências e porcentagens da origem do encaminhamento por ano em que ocorreu o primeiro atendimento

\begin{tabular}{|c|c|c|c|c|c|c|c|}
\hline \multirow{2}{*}{ Encaminhamento } & \multicolumn{6}{|c|}{ Ano $1^{\circ}$ atendimento } & \multirow{2}{*}{ Total } \\
\hline & 2010 & 2011 & 2012 & 2013 & 2014 & 2015 & \\
\hline \multirow[t]{2}{*}{ INSTITUIÇÕES } & 3 & 4 & 4 & 5 & 6 & 4 & 28 \\
\hline & $3,70 \%$ & $2,80 \%$ & $2,40 \%$ & $2,70 \%$ & $3,10 \%$ & $4,30 \%$ & $3,30 \%$ \\
\hline \multirow[t]{2}{*}{ AE/AMA/AME } & 9 & 13 & 7 & 5 & 9 & 2 & 46 \\
\hline & $12,30 \%$ & $9,20 \%$ & $4,20 \%$ & $2,70 \%$ & $4,80 \%$ & $2,20 \%$ & $5,40 \%$ \\
\hline \multirow[t]{2}{*}{$\begin{array}{c}\text { CAPS/CER/NISA/NIR/ } \\
\text { UBS }\end{array}$} & 18 & 40 & 62 & 74 & 88 & 55 & 360 \\
\hline & $22,20 \%$ & $28,40 \%$ & $49,90 \%$ & $40,80 \%$ & $45,80 \%$ & $58,10 \%$ & $41,90 \%$ \\
\hline \multirow[t]{2}{*}{ Demanda Espontânea } & 4 & 3 & 4 & 5 & 2 & 1 & 19 \\
\hline & $4,90 \%$ & $2,10 \%$ & $2,40 \%$ & $2,70 \%$ & $1,10 \%$ & $1,10 \%$ & $2,20 \%$ \\
\hline \multirow[t]{2}{*}{ Escola } & 1 & 1 & 0 & 1 & 1 & 0 & 4 \\
\hline & $1,20 \%$ & $0,70 \%$ & $0,00 \%$ & $0,50 \%$ & $0,50 \%$ & $0,00 \%$ & $0,50 \%$ \\
\hline \multirow[t]{2}{*}{ Hospital } & 4 & 10 & 9 & 5 & 4 & 1 & 33 \\
\hline & $4,90 \%$ & $7,10 \%$ & $5,50 \%$ & $2,70 \%$ & $2,10 \%$ & $1,10 \%$ & $3,90 \%$ \\
\hline \multirow[t]{2}{*}{ Maternidade } & 27 & 38 & 31 & 62 & 59 & 21 & 238 \\
\hline & $33,30 \%$ & $27,00 \%$ & $18,90 \%$ & $33,30 \%$ & $31,10 \%$ & $22,10 \%$ & $27,80 \%$ \\
\hline \multirow[t]{2}{*}{ Outros } & 13 & 32 & 27 & 26 & 21 & 11 & 129 \\
\hline & $16,0 \%$ & $22,7 \%$ & $16,5 \%$ & $13,4 \%$ & $11,1 \%$ & $11,6 \%$ & $15,1 \%$ \\
\hline \multirow[t]{2}{*}{ Total } & 81 & 141 & 164 & 186 & 190 & 95 & 857 \\
\hline & $100,0 \%$ & $100,0 \%$ & $100,0 \%$ & $100,0 \%$ & $100,0 \%$ & $100,0 \%$ & $100,0 \%$ \\
\hline
\end{tabular}

Legenda: INSTITUIÇÕES - foram consideradas APAE (Associação de Pais e Amigos dos Excepcionais), AACD (Associação de Apoio a Criança Deficiente) e CEMA (Centro de Medicina Avançado); AE (ambulatório de especialidades); AMA (Assistência Médica Ambulatorial); AME (Ambulatório Médico de Especialidades); CAPS (Centro de Atenção Psicossocial); CER (Centro Especializado em Reabilitação); NIR (Núcleo Integrado em Reabilitação); NISA (Núcleo Integrado em Saúde Auditiva); UBS (Unidade Básica de Saúde) 
A principal queixa para busca do diagnóstico foi a falha na TANU nas maternidades e suspeita de perdas auditivas na primeira infância.

Também vale ressaltar que, em todos os anos, a maior parte das crianças atendidas possuía algum tipo de IRDA. Estas crianças têm maior probabili- dade de terem uma deficiência auditiva, e são mais observadas no seu desenvolvimento, pela preocupação dos pais e profissionais de saúde.

A faixa etária no diagnóstico, predominante em todos os anos, foi acima de 12 meses de idade, seguida de crianças com até 3 meses de vida.

Tabela 4. Distribuições de frequências e porcentagens de respostas a "fez tanu" por ano em que ocorreu o primeiro atendimento

\begin{tabular}{|c|c|c|c|c|}
\hline \multirow{2}{*}{ Ano $1^{\circ}$ atendimento } & \multicolumn{3}{|c|}{ Fez TANU } & \multirow{2}{*}{ Total } \\
\hline & $\mathbf{N}$ & $\mathbf{S}$ & SD & \\
\hline \multirow[t]{2}{*}{2010} & 15 & 41 & 25 & 81 \\
\hline & $18,5 \%$ & $50,6 \%$ & $30,9 \%$ & $100,0 \%$ \\
\hline \multirow[t]{2}{*}{2011} & 15 & 75 & 51 & 141 \\
\hline & $10,6 \%$ & $53,2 \%$ & $36,2 \%$ & $100,0 \%$ \\
\hline \multirow[t]{2}{*}{2012} & 17 & 85 & 62 & 164 \\
\hline & $10,4 \%$ & $51,8 \%$ & $37,8 \%$ & $100,0 \%$ \\
\hline \multirow{2}{*}{2013} & 13 & 116 & 57 & 186 \\
\hline & $7,0 \%$ & $62,4 \%$ & $30,6 \%$ & $100,0 \%$ \\
\hline \multirow[t]{2}{*}{2014} & 11 & 122 & 57 & 190 \\
\hline & $5,8 \%$ & $64,2 \%$ & $30,0 \%$ & $100,0 \%$ \\
\hline \multirow[t]{2}{*}{2015} & 6 & 56 & 33 & 95 \\
\hline & $6,3 \%$ & $58,9 \%$ & $34,7 \%$ & $100,0 \%$ \\
\hline \multirow[t]{2}{*}{ Total } & 77 & 495 & 285 & 857 \\
\hline & $9,0 \%$ & $57,8 \%$ & $33,3 \%$ & $100,0 \%$ \\
\hline
\end{tabular}

Legenda: $\mathrm{N}=\mathrm{Não} ; \mathrm{S}=\mathrm{Sim} ; \mathrm{SD}=\mathrm{Sem}$ dados

Tabela 5. Distribuição de frequências e porcentagens do motivo para procura de exames audiológicos, por ano que ocorreu o primeiro atendimento

\begin{tabular}{|c|c|c|c|c|c|c|}
\hline \multirow{2}{*}{ ano $1^{\circ}$ atendimento } & \multicolumn{5}{|c|}{ Motivo encaminhamento } & \multirow{2}{*}{ Total } \\
\hline & Auditiva & DNPM & Fala/Lgg & Prótese & Triagem & \\
\hline \multirow[t]{2}{*}{2010} & 45 & 0 & 6 & 0 & 30 & 81 \\
\hline & $55,60 \%$ & $0,00 \%$ & $7,40 \%$ & $0,00 \%$ & $37,00 \%$ & $100,00 \%$ \\
\hline \multirow[t]{2}{*}{2011} & 93 & 3 & 11 & 1 & 33 & 141 \\
\hline & $66,00 \%$ & $2,10 \%$ & $7,80 \%$ & $0,70 \%$ & $23,40 \%$ & $100,00 \%$ \\
\hline \multirow[t]{2}{*}{2012} & 104 & 1 & 24 & 0 & 35 & 164 \\
\hline & $63,40 \%$ & $0,60 \%$ & $14,60 \%$ & $0,00 \%$ & $21,30 \%$ & $100,00 \%$ \\
\hline \multirow[t]{2}{*}{2013} & 98 & 1 & 25 & 0 & 62 & 186 \\
\hline & $52,70 \%$ & $50,00 \%$ & $13,40 \%$ & $0,00 \%$ & $33,30 \%$ & $100,00 \%$ \\
\hline \multirow[t]{2}{*}{2014} & 81 & 5 & 33 & 8 & 63 & 190 \\
\hline & $42,60 \%$ & $2,60 \%$ & $17,40 \%$ & $4,20 \%$ & $33,20 \%$ & $100,00 \%$ \\
\hline \multirow[t]{2}{*}{2015} & 49 & 3 & 11 & 1 & 31 & 95 \\
\hline & $51,60 \%$ & $3,20 \%$ & $11,60 \%$ & $1,10 \%$ & $33,60 \%$ & $100,00 \%$ \\
\hline \multirow[t]{2}{*}{ TOTAL } & 470 & 13 & 110 & 10 & 254 & 857 \\
\hline & $54,80 \%$ & $1,50 \%$ & $12,80 \%$ & $1,20 \%$ & $29,60 \%$ & $100,00 \%$ \\
\hline
\end{tabular}

Legenda: DNPM = Desenvolvimento Neuro Psico Motor; Lgg = linguagem 
Tabela 6. Distribuições de frequências e porcentagens de crianças com IRDA por ano em que ocorreu o primeiro atendimento

\begin{tabular}{|c|c|c|c|c|}
\hline \multirow{2}{*}{$\begin{array}{c}\text { Ano } 1^{\circ} \\
\text { atendimento }\end{array}$} & \multicolumn{3}{|c|}{ IRDA } & \multirow{2}{*}{ Total } \\
\hline & $\mathbf{N}$ & $\mathbf{S}$ & SD & \\
\hline \multirow[t]{2}{*}{2010} & 25 & 55 & 1 & 81 \\
\hline & $30,9 \%$ & $67,9 \%$ & $1,2 \%$ & $100,0 \%$ \\
\hline \multirow[t]{2}{*}{2011} & 50 & 90 & 1 & 141 \\
\hline & $35,5 \%$ & $63,8 \%$ & $0,7 \%$ & $100,0 \%$ \\
\hline \multirow[t]{2}{*}{2012} & 54 & 110 & 0 & 164 \\
\hline & $32,9 \%$ & $67,1 \%$ & $0,0 \%$ & $100,0 \%$ \\
\hline \multirow[t]{2}{*}{2013} & 63 & 123 & 0 & 186 \\
\hline & $33,9 \%$ & $66,1 \%$ & $0,0 \%$ & $100,0 \%$ \\
\hline \multirow[t]{2}{*}{2014} & 62 & 126 & 2 & 190 \\
\hline & $32,6 \%$ & $66,3 \%$ & $1,1 \%$ & $100,0 \%$ \\
\hline \multirow[t]{2}{*}{2015} & 37 & 57 & 1 & 95 \\
\hline & $38,9 \%$ & $60,0 \%$ & $1,1 \%$ & $100,0 \%$ \\
\hline \multirow[t]{2}{*}{ Total } & 291 & 561 & 5 & 857 \\
\hline & $34,0 \%$ & $65,5 \%$ & $0,6 \%$ & $100,0 \%$ \\
\hline
\end{tabular}

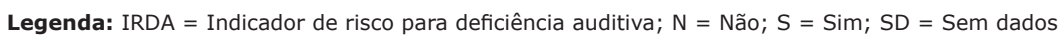

Tabela 7. Distribuição de frequências e porcentagens da faixa etária do diagnóstico por ano.

\begin{tabular}{|c|c|c|c|c|c|}
\hline \multirow{2}{*}{$\begin{array}{c}\text { ano } 1^{\circ} \\
\text { atendimento }\end{array}$} & \multicolumn{4}{|c|}{ Faixa etária no diagnóstico } & \multirow[b]{2}{*}{ Total } \\
\hline & 0 a 3 meses & 4 a 6 meses & 7 a 12 meses & $\begin{array}{c}\text { mais que } 12 \\
\text { meses }\end{array}$ & \\
\hline \multirow[t]{2}{*}{2010} & 23 & 8 & 13 & 37 & 81 \\
\hline & $28,40 \%$ & $9,90 \%$ & $16,00 \%$ & $45,70 \%$ & $100,00 \%$ \\
\hline \multirow{2}{*}{2011} & 23 & 22 & 24 & 72 & 141 \\
\hline & $16,30 \%$ & $15,60 \%$ & $17,00 \%$ & $51,10 \%$ & $100,00 \%$ \\
\hline \multirow[t]{2}{*}{2012} & 19 & 10 & 40 & 95 & 164 \\
\hline & $11,60 \%$ & $6,10 \%$ & $24,40 \%$ & $57,90 \%$ & $100,00 \%$ \\
\hline \multirow[t]{2}{*}{2013} & 37 & 34 & 26 & 89 & 186 \\
\hline & $19,90 \%$ & $18,30 \%$ & $14,00 \%$ & $47,80 \%$ & $100,00 \%$ \\
\hline \multirow[t]{2}{*}{2014} & 39 & 25 & 39 & 87 & 190 \\
\hline & $20,50 \%$ & $13,20 \%$ & $20,50 \%$ & $45,80 \%$ & $100,00 \%$ \\
\hline \multirow[t]{2}{*}{2015} & 17 & 9 & 19 & 50 & 95 \\
\hline & $17,90 \%$ & $9,50 \%$ & $20,00 \%$ & $52,60 \%$ & $100,00 \%$ \\
\hline \multirow[t]{2}{*}{ TOTAL } & 158 & 108 & 161 & 430 & 857 \\
\hline & $18,40 \%$ & $12,60 \%$ & $18,80 \%$ & $50,20 \%$ & $100,00 \%$ \\
\hline
\end{tabular}


A duração do diagnóstico variou entre 02 e 06 meses.

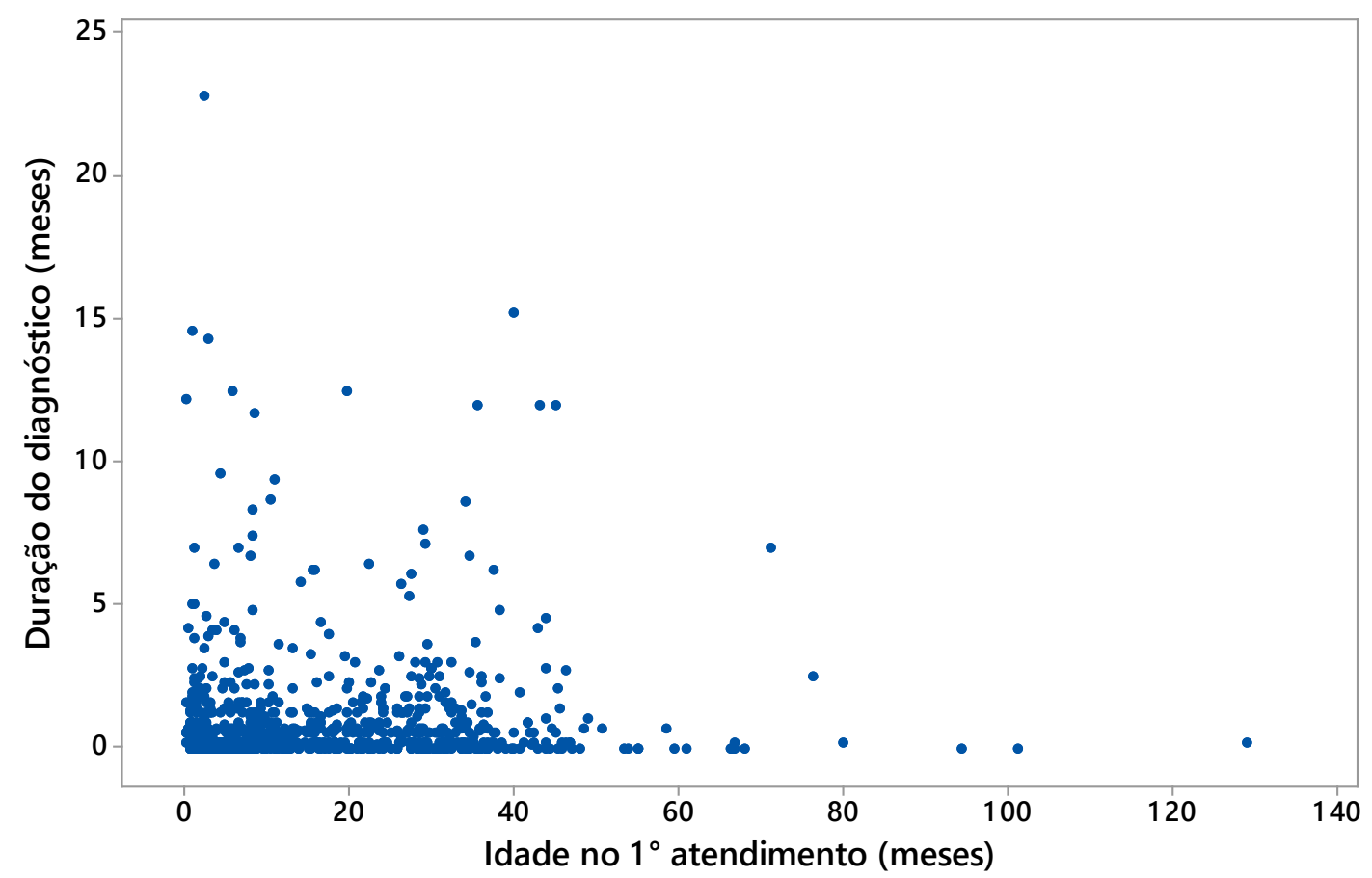

Figura 1. Diagrama de dispersão do tempo de duração do diagnóstico e a idade no primeiro atendimento

Com exceção dos anos de 2012 e 2015, o número de crianças que fizeram TANU e concluíram o diagnóstico até os 03 meses de idade, foi maior que as outras faixas etárias. Já relacionando o resultado da TANU e o diagnóstico, pode-se observar que as falhas obtidas na triagem foram confirmadas no diagnóstico, mostrando a eficiência desses procedimentos para a identificação de perdas auditivas, em idade considerada ideal para o início da reabilitação. Mostra-se, mais uma vez, a importância deste procedimento simples, rápido e barato, para o início precoce da intervenção necessária.
Houve concordância entre os tipos de perda nas duas orelhas na maioria das crianças, ou seja, a caracterização audiológica mostra uma simetria entre as orelhas, no que se refere à perda auditiva.

Apresenta-se ainda a relação entre idade do diagnóstico e IRDA, mostrando que crianças com IRDA têm maior probabilidade de terem perdas auditivas e são acompanhadas de perto, sendo encaminhadas mais cedo aos centros de referência. 
Tabela 8. Distribuições de frequências e porcentagens quanto à resposta "fez TANU" em cada combinação do diagnóstico nas duas orelhas

\begin{tabular}{|c|c|c|c|c|}
\hline \multirow{2}{*}{ Diagnóstico OD/OE } & \multicolumn{3}{|c|}{ Fez TANU } & \multirow{2}{*}{ Total } \\
\hline & $\mathbf{N}$ & $\mathbf{S}$ & SD & \\
\hline \multirow[t]{2}{*}{ Normal/Normal } & 44 & 198 & 146 & 388 \\
\hline & $11,3 \%$ & $51,0 \%$ & $37,6 \%$ & $100,0 \%$ \\
\hline \multirow{2}{*}{ Normal/Condutiva } & 2 & 17 & 12 & 31 \\
\hline & $6,5 \%$ & $54,8 \%$ & $38,7 \%$ & $100,0 \%$ \\
\hline \multirow[t]{2}{*}{ Normal/Mista } & 1 & 1 & 0 & 2 \\
\hline & $50,0 \%$ & $50,0 \%$ & $0,0 \%$ & $100,0 \%$ \\
\hline \multirow[t]{2}{*}{ Normal/Sensórioneural } & 0 & 8 & 2 & 10 \\
\hline & $0,0 \%$ & $80,0 \%$ & $20,0 \%$ & $100,0 \%$ \\
\hline \multirow[t]{2}{*}{ Condutiva/Condutiva } & 14 & 100 & 38 & 152 \\
\hline & $9,2 \%$ & $65,8 \%$ & $25,0 \%$ & $100,0 \%$ \\
\hline \multirow[t]{2}{*}{ Condutiva/Mista } & 0 & 2 & 0 & 2 \\
\hline & $0,0 \%$ & $100,0 \%$ & $0,0 \%$ & $100,0 \%$ \\
\hline \multirow[t]{2}{*}{ Condutiva/Normal } & 6 & 34 & 7 & 47 \\
\hline & $12,8 \%$ & $72,3 \%$ & $14,9 \%$ & $100,0 \%$ \\
\hline \multirow[t]{2}{*}{ Condutiva/Sensórioneural } & 0 & 1 & 1 & 2 \\
\hline & $0,0 \%$ & $50,0 \%$ & $50,0 \%$ & $100,0 \%$ \\
\hline \multirow[t]{2}{*}{ Mista/Mista } & 0 & 11 & 5 & 16 \\
\hline & $0,0 \%$ & $68,8 \%$ & $31,3 \%$ & $100,0 \%$ \\
\hline \multirow[t]{2}{*}{ Mista/Normal } & 0 & 1 & 0 & 1 \\
\hline & $0,0 \%$ & $100,0 \%$ & $0,0 \%$ & $100,0 \%$ \\
\hline \multirow[t]{2}{*}{ Mista/Sensórioneural } & 0 & 1 & 0 & 1 \\
\hline & $0,0 \%$ & $100,0 \%$ & $0,0 \%$ & $100,0 \%$ \\
\hline \multirow[t]{2}{*}{ Sensórioneural/Condutiva } & 0 & 3 & 0 & 3 \\
\hline & $0,0 \%$ & $100,0 \%$ & $0,0 \%$ & $100,0 \%$ \\
\hline \multirow[t]{2}{*}{ Sensórioneural/Normal } & 1 & 7 & 2 & 10 \\
\hline & $10,0 \%$ & $70,0 \%$ & $20,0 \%$ & $100,0 \%$ \\
\hline \multirow[t]{2}{*}{ Sensórioneural/Sensórioneural } & 9 & 111 & 72 & 192 \\
\hline & $4,7 \%$ & $57,8 \%$ & $37,5 \%$ & $100,0 \%$ \\
\hline \multirow[t]{2}{*}{ Total } & 77 & 495 & 285 & 857 \\
\hline & $9,0 \%$ & $57,8 \%$ & $33,3 \%$ & $100,0 \%$ \\
\hline
\end{tabular}

Tabela 9. Distribuições de frequências e porcentagens conjuntas e marginais do tipo de perda auditiva por orelha

\begin{tabular}{|c|c|c|c|c|c|}
\hline \multirow{2}{*}{ Tipo de perda OD } & \multicolumn{4}{|c|}{ Tipo de perda OE } & \multirow[b]{2}{*}{ Total } \\
\hline & Normal & Condutiva & Mista & Sensórioneural & \\
\hline \multirow[t]{2}{*}{ Normal } & 388 & 31 & 2 & 10 & 431 \\
\hline & $45,3 \%$ & $3,6 \%$ & $0,2 \%$ & $1,2 \%$ & $50,3 \%$ \\
\hline \multirow[t]{2}{*}{ Condutiva } & 47 & 152 & 2 & 2 & 203 \\
\hline & $5,5 \%$ & $17,7 \%$ & $0,2 \%$ & $0,2 \%$ & $23,7 \%$ \\
\hline \multirow[t]{2}{*}{ Mista } & 1 & 0 & 16 & 1 & 18 \\
\hline & $0,1 \%$ & $0,0 \%$ & $1,9 \%$ & $0,1 \%$ & $2,1 \%$ \\
\hline \multirow[t]{2}{*}{ Sensórioneural } & 10 & 3 & 0 & 192 & 205 \\
\hline & $1,2 \%$ & $0,4 \%$ & $0,0 \%$ & $22,4 \%$ & $23,9 \%$ \\
\hline \multirow[t]{2}{*}{ Total } & 446 & 186 & 20 & 205 & 857 \\
\hline & $52,0 \%$ & $21,7 \%$ & $2,3 \%$ & $23,9 \%$ & $100,0 \%$ \\
\hline
\end{tabular}


Tabela 10. Distribuições de frequências e porcentagens da faixa etária no diagnóstico em relação a possuir IRDA por ano em que ocorreu o primeiro atendimento

\begin{tabular}{|c|c|c|c|c|c|c|}
\hline \multicolumn{7}{|c|}{ Idade no diagnóstico } \\
\hline $\begin{array}{c}\text { Ano10 }^{\circ} \\
\text { atendimento }\end{array}$ & IRDA & 0 a 3 meses & 4 a 6 meses & 7 a 12 meses & $\begin{array}{c}\text { mais que } 12 \\
\text { meses }\end{array}$ & Total \\
\hline \multirow[t]{6}{*}{2010} & $\mathrm{~N}$ & 8 & 3 & 1 & 13 & 25 \\
\hline & & $32,0 \%$ & $12,0 \%$ & $4,0 \%$ & $52,0 \%$ & $100,0 \%$ \\
\hline & $\mathrm{S}$ & 15 & 5 & 12 & 23 & 55 \\
\hline & & $27,3 \%$ & $9,1 \%$ & $21,8 \%$ & $41,8 \%$ & $100,0 \%$ \\
\hline & Total & 23 & 8 & 13 & 36 & 80 \\
\hline & & $28,7 \%$ & $10,0 \%$ & $16,3 \%$ & $45,0 \%$ & $100,0 \%$ \\
\hline \multirow[t]{6}{*}{2011} & $\mathrm{~N}$ & 11 & 7 & 8 & 24 & 50 \\
\hline & & $22,0 \%$ & $14,0 \%$ & $16,0 \%$ & $48,0 \%$ & $100,0 \%$ \\
\hline & S & 12 & 15 & 16 & 47 & 90 \\
\hline & & $13,3 \%$ & $16,7 \%$ & $17,8 \%$ & $52,2 \%$ & $100,0 \%$ \\
\hline & Total & 23 & 22 & 24 & 71 & 140 \\
\hline & & $16,4 \%$ & $15,7 \%$ & $17,1 \%$ & $50,7 \%$ & $100,0 \%$ \\
\hline \multirow[t]{6}{*}{2012} & $\mathrm{~N}$ & 8 & 3 & 10 & 33 & 54 \\
\hline & & $14,8 \%$ & $5,6 \%$ & $18,5 \%$ & $61,1 \%$ & $100,0 \%$ \\
\hline & S & 11 & 7 & 30 & 62 & 110 \\
\hline & & $10,0 \%$ & $6,4 \%$ & $27,3 \%$ & $56,4 \%$ & $100,0 \%$ \\
\hline & Total & 19 & 10 & 40 & 95 & 164 \\
\hline & & $11,6 \%$ & $6,1 \%$ & $24,4 \%$ & $57,9 \%$ & $100,0 \%$ \\
\hline \multirow[t]{6}{*}{2013} & $\mathrm{~N}$ & 14 & 9 & 6 & 34 & 63 \\
\hline & & $22,2 \%$ & $14,3 \%$ & $9,5 \%$ & $54,0 \%$ & $100,0 \%$ \\
\hline & S & 23 & 25 & 20 & 55 & 123 \\
\hline & & $18,7 \%$ & $20,3 \%$ & $16,3 \%$ & $44,7 \%$ & $100,0 \%$ \\
\hline & Total & 37 & 34 & 26 & 89 & 186 \\
\hline & & $19,9 \%$ & $18,3 \%$ & $14,0 \%$ & $47,8 \%$ & $100,0 \%$ \\
\hline \multirow[t]{6}{*}{2014} & $\mathrm{~N}$ & 15 & 5 & 17 & 25 & 62 \\
\hline & & $24,2 \%$ & $8,1 \%$ & $27,4 \%$ & $40,3 \%$ & $100,0 \%$ \\
\hline & S & 24 & 19 & 22 & 61 & 126 \\
\hline & & $19,0 \%$ & $15,1 \%$ & $17,5 \%$ & $48,4 \%$ & $100,0 \%$ \\
\hline & Total & 39 & 24 & 39 & 86 & 188 \\
\hline & & $20,7 \%$ & $12,8 \%$ & $20,7 \%$ & $45,7 \%$ & $100,0 \%$ \\
\hline \multirow[t]{6}{*}{2015} & $\mathrm{~N}$ & 7 & 2 & 9 & 19 & 37 \\
\hline & & $18,9 \%$ & $5,4 \%$ & $24,3 \%$ & $51,4 \%$ & $100,0 \%$ \\
\hline & S & 10 & 6 & 10 & 31 & 57 \\
\hline & & $17,5 \%$ & $10,5 \%$ & $17,5 \%$ & $54,4 \%$ & $100,0 \%$ \\
\hline & Total & 17 & 8 & 19 & 50 & 94 \\
\hline & & $18,1 \%$ & $8,5 \%$ & $20,2 \%$ & $53,2 \%$ & $100,0 \%$ \\
\hline
\end{tabular}

\section{Discussão}

Os resultados demonstrados na Tabela 1 apontam que a idade no primeiro atendimento, em sua maioria, ocorreu por volta dos 12 meses de idade.

A literatura recomenda que o diagnóstico seja concluído até o terceiro mês de vida, o que não ocorre na maioria das crianças inseridas no presente estudo $^{5,6,7}$. Tais recomendações são ratificadas por estudos que relatam que o processo de maturação da parte periférica da via auditiva até o tronco encefálico ocorre ainda na fase intrauterina, e após o nascimento, com a mielinização das vias auditivas, completando-se este processo inicial por volta dos
18 meses de vida. Sabe-se, no entanto, que a privação sensorial, pode prejudicar o amadurecimento dessas vias, interferindo nas formações das redes neurais e suas conexões com o córtex auditivo. Portanto, o diagnóstico precoce, nos primeiros meses de vida, é necessário para melhor aproveitamento do resíduo auditivo e da plasticidade neuronal, e consequentemente, um bom desenvolvimento da linguagem $1,2,3,4,8,9,16$.

A faixa etária prevalente, de acordo com a Tabela 01, foi de crianças acima de 12 meses de idade. Apenas $23,1 \%$ das crianças estavam na faixa etária abaixo de 03 meses de idade. No ano de 2010, com o início da obrigatoriedade da TANU 
em todo território nacional, por meio de lei federal, esperava-se uma diminuição da faixa etária que compareceria para diagnóstico, após falha na TANU. O presente estudo, no período de 2010 a 2015 , pode ter mostrado resultados que foram influenciados pela força da lei publicada em 2010, porém, ainda sem todos os efeitos desejados para o diagnóstico precoce, após 5 anos de implantação da TANU no município. Assim, a maioria das crianças no presente estudo, ainda iniciou o processo de diagnóstico audiológico após os 12 meses de vida. Entende-se que, apesar da obrigatoriedade da TANU, nem todas as maternidades do município a realizam conforme recomendações nacionais e internacionais e, principalmente, questiona-se se o sistema de saúde está organizado para encaminhar todas as crianças com falha na TANU para o diagnóstico especializado. Este não ocorre com eficiência em todas as maternidades, sendo um indicador de qualidade a ser estudado ${ }^{10,17}$.

A região Sul (64,2\%), seguida da Norte $(21,6 \%)$, identificadas como região de moradia, foram predominantes no encaminhamento para diagnóstico (Tabela 02). Este dado ocorre devido ao princípio de regionalização do Sistema Único de Saúde (SUS). O CeAC é credenciado como um serviço de referência em Saúde Auditiva, preparado para receber crianças para diagnóstico audiológico, de qualquer faixa etária, e denominado como Centro Especializado em Reabilitação em duas modalidades, auditiva e intelectual (CERII). As duas regiões em questão têm como referência a instituição que foi local do estudo. A regionalização é princípio do SUS, como forma de organização dos serviços e facilitação do acesso aos cidadãos. É importante que o acesso ao atendimento seja mais próximo à residência, auxiliando na detecção precoce, diagnóstico, tratamento e reabilitação ${ }^{11,12,18}$.

Os encaminhamentos que mais se destacaram foram das maternidades, seguido de outros CER e do Núcleo Integrado de Saúde Auditiva (NISA) (Tabela 3). O CER e o NISA fazem parte da Rede de Atenção à Saúde Auditiva do município e, quando não conseguem resolver todas as demandas do paciente, encaminham para serviços de maior complexidade como o CeAC. Sendo assim, tal resultado mostra que a rede de serviços em saúde auditiva está funcionando conforme preconiza o SUS, ou seja, de forma regionalizada e hierarquizada ${ }^{11}$.

Os serviços de atenção à saúde auditiva vêm recebendo crianças para diagnóstico e/ou interven- ção, ainda de forma tardia, mas o número expressivo no acompanhamento, mostra um processo de reabilitação mais efetivo. Na Tabela 1 podemos ver que de 2012 para 2014 ocorreu uma duplicação do número de crianças que iniciou o diagnóstico antes dos 3 meses de vida, embora esse grupo ainda seja menor do que aquele de crianças com 12 meses de vida ou mais. Isto reflete que, com a obrigatoriedade da TANU, a partir de agosto de 2010, houve melhora na identificação precoce da perda auditiva e, posteriormente, na reabilitação precoce, diminuindo, portanto, os efeitos da deficiência auditiva sobre a criança. ${ }^{8,10,18}$.

Esses dados corroboram com os achados de um estudo na Itália, no qual os autores identificaram que a idade média do diagnóstico, no Centro de Audiologia e Foniatria de Turin, era de 20,5 meses de idade; mas quando considerado somente o grupo de crianças que realizaram a TANU, esta idade diminuía para 6.8 meses, demonstrando que a estratégia da TANU leva a um diagnóstico precoce de alterações auditivas ${ }^{19}$.

A maioria das crianças que compareceu para avaliação audiológica, realizou a TANU $(57,8 \%)$, porém, este número deveria ser $100 \%$ de crianças triadas, uma vez que a lei que versa sobre a obrigatoriedade da realização desse exame existe desde 2010, em todo território nacional, e abrange crianças com ou sem IRDA (Tabela 4). Com isso, pode-se inferir que ainda há locais que não cumprem a determinação da lei, prejudicando o diagnóstico e intervenção precoces. A conscientização dos pais e responsáveis, e o esclarecimento à sociedade civil tornam-se fatores importantes quanto à exigência e realização da TANU ${ }^{10,20,21}$. Vale ressaltar que uma política nacional, que regulamente a realização da TANU e seus subsequentes processos de diagnóstico e intervenção ainda não foi publicada pelo Ministério da Saúde. Alguns autores citam ainda, fatores que podem atrasar o diagnóstico de alterações auditivas, tais como o baixo nível sócio econômico da população e o pouco conhecimento dos sintomas e formas de reabilitação da deficiência auditiva $^{22}$.

Pesquisa realizada na Turquia, com análise retrospectiva de 199 prontuários no período entre 1999 e 2004, e 156 prontuários entre 1991 e 1994 , mostra que a idade da suspeita, identificação e intervenção foram estatisticamente menores no grupo de 1999 a 2004, quando comparado com o grupo de anos anteriores. Apesar disso, somente $8,5 \%$ dos 
casos foi identificado antes dos 6 meses de idade, e somente 1\% iniciou intervenção até os 6 meses. Concluíram que a TANU deve ser implantada para diminuir a idade de identificação e intervenção de alterações auditivas ${ }^{22}$.

A idade no diagnóstico foi em média doze meses de idade, e que para esses indivíduos, os motivos dos encaminhamentos foram por suspeita de alterações auditivas ou atraso de fala e linguagem $^{19,23}$.

No Brasil, em um estudo realizado em um centro em Jundiaí, foram analisados, retrospectivamente, 313 prontuários de crianças. Também nesse estudo, foi observado que a idade do diagnóstico, intervenção e indicação de dispositivos de amplificação foi menor quando as crianças foram encaminhadas após a falha na TANU. Houve vantagem de 40,7 meses no diagnóstico, 45,8 meses na intervenção e 54,9 meses na indicação de dispositivos de amplificação, quando comparados com crianças advindas de outros profissionais/locais, que não das maternidades que realizam TANU. Além disso, analisaram que a demora em concluir o diagnóstico, de algumas crianças advindas da TANU, foi devido a não adesão das famílias que faltaram em consultas, além de dificuldades na conclusão precisa do diagnóstico audiológico. Esta avaliação em neonatos e lactentes exige procedimentos específicos, como PEATE com Frequência Específica (PEATE-FE), tanto por via aérea, como por via óssea. Tais exames demandam profissionais experientes e capacitados na realização destes ${ }^{24}$.

As crianças que compareceram para diagnóstico após falha da TANU (Tabela 8) concluem o diagnóstico da perda auditiva mais precocemente, por volta do $3^{\circ}$ mês de vida. Mesmo em países considerados desenvolvidos, a idade média para o diagnóstico da perda auditiva ainda é considerada alta, com estudos indicando idade média de 5,4 anos ${ }^{23}$.

O presente estudo revela que $65,5 \%$ das crianças apresentaram algum tipo de IRDA (Tabela 6). Alguns estudos citam a correlação entre perda auditiva e IRDA, revelando com ocorrência maior de alterações auditivas entre o grupo que possui um ou mais IRDA. 25,26,27,28.

Em um estudo no Brasil, foi constatado que $0,6 \%$ das crianças que falharam na TANU apresentavam um ou mais IRDA. A ocorrência de falha na TANU foi menor em crianças com até um IRDA quando comparadas com outras com mais indicadores. Houve associação estatisticamente significante entre a ocorrência de falha e o número de IRDA. Também demonstraram que, o índice de falha em crianças prematuras e de muito baixo peso, foi maior do que nos grupos com nascimento a termo e prematuras com peso maior que $1500 \mathrm{~g}$. Este fato indica que as crianças do primeiro grupo estão mais vulneráveis a ficarem internadas em Unidade de Terapia Intensiva Neonatal (UTIN), com maior probabilidade de receberem medicações ototóxicas, além da exposição à ventilação mecânica. Das crianças que falharam, $0,5 \%$ apresentaram alterações auditivas, sendo que a frequência de Deficiência Auditiva (DA) foi maior em crianças prematuras quando comparadas a crianças nascidas a termo; e maior entre prematuros de muito baixo peso quando comparados a prematuros com peso superior a $1500 \mathrm{~g}$ no nascimento. Também houve maior ocorrência de DA em crianças com um ou mais IRDA, mas relatam que ocorreu um caso de DA em criança sem IRDA, o que reforça a importância da TANU, ou seja, em todas as crianças e não somente naquelas com histórico que apontem para IRDA ${ }^{28}$.

A relação entre IRDA e o diagnóstico (Tabela 10), mostra que a maioria das crianças possui IRDA $(65,5 \%)$. O indicador de risco mais frequente foi a permanência na UTIN, por mais de 5 dias, seguido de antecedentes familiares de deficiência auditiva. Alguns estudos apontam essa correlação entre perda auditiva e IRDA, revelando a ocorrência maior entre o grupo que possui um ou mais indicadores. Destacam a permanência em UTIN como fator de risco frequente entre a população estudada ${ }^{21,23}$, 28,29,30,31.

Em relação aos IRDA, alguns autores relatam que há maior incidência de DA em países em desenvolvimento devido a piores condições de saúde, sócio econômicas, alto índice de mortalidade infantil, privação de adequado pré-natal, calculando-se que a incidência deve ser de 6 para 1000 bebês. Referem que doenças, como o sarampo, meningite, caxumba e rubéola, devem ser prevenidas por meio de vacinas, nas ações de atenção básica. Indicam ainda que, melhores práticas obstétricas, neonatais e de cuidados com a criança devem ocorrer para minimizar a anóxia neonatal, prematuridade e crianças com baixo peso no nascimento. Propõem a inclusão de ações na atenção básica, de promoção à saúde materno infantil, ações de educação em saúde e conscientização quanto a doenças como citome- 
galus vírus, toxoplasmose, herpes e sífilis, além do cuidado da gestante, identificando e tratando sífilis e toxoplasmose gestacional ${ }^{32}$.

A audição dentro da normalidade, em ambas as orelhas, foi o diagnóstico obtido, no presente estudo, na maioria das crianças. Porém, as perdas mistas e sensórioneurais somaram $26 \%$ na orelha direita e $26,2 \%$ na orelha esquerda (Tabela 09). Estudos de prevalência de perdas auditivas referem que a perda condutiva aparece em número maior, seguida das perdas sensórioneurais e mistas. Esse número é considerado elevado, se comparado com países desenvolvidos, o que sugere mais estudos e investimentos, para promoção à saúde e prevenção da surdez ${ }^{25,29}$.

Ressalta-se que houve predominância de início do diagnóstico para crianças com mais de 12 meses de idade. Isso demostra como a importância da política de saúde auditiva é essencial para que essas crianças cheguem o mais cedo possível para um diagnóstico precoce, não perdendo, portanto, um tempo essencial para o desenvolvimento da linguagem. Infelizmente, a implantação da PNASA não é uniforme no território nacional. Deste modo, os direitos de integralidade e universalidade ainda não estão garantidos a todos ${ }^{11,13,14,15}$.

\section{Conclusão}

A partir deste estudo, realizado com os dados disponíveis no CeAC, de agosto de 2010 a junho de 2015, conclui-se: a regionalização do serviço de saúde tem sido alcançada, uma vez que as regiões da área de referência são as que mais encaminham para diagnóstico; as queixas auditivas são as que têm maior ocorrência na busca por exames audiológicos, em especial para crianças com IRDA em suas histórias; em todos os anos estudados houve crianças que chegaram para o primeiro atendimento com menos de 01 mês de idade, porém em número reduzido; a faixa etária prevalente no primeiro atendimento foi de crianças acima de 12 meses de idade, o que mostra que o diagnóstico precoce ainda não tem sido alcançado em sua maioria; as perdas auditivas sensórioneurais profundas foram as mais prevalentes, talvez pela maior visibilidade entre pais e profissionais da saúde; as políticas públicas de TANU devem ser publicadas e implantadas, de forma mais sistemática, para que se alcance o diagnóstico e a intervenção precoce.

\section{Referências bibliográficas}

1. JAMIESON, JR. O impacto da deficiência auditiva. In: Katzz, J. Tratado de audiologia Clínica. São Paulo: Manole; 1999. p. 590-609.

2. Joint Committee on Infant Hearing. Year 2000 Position Statement. Principles and guidelines for early detection and intervention programs. Am J Audiol .2000; 9(1): 9-29.

3. Yoshinaga-Itano C. From screening to early identification and intervention: discovering predictor to successful outcomes for children with significant hearing loss. J Deaf Stud Deaf Educ. 2003, 8(1): 11-30;

4. Brasil. Ministério da Saúde. Portaria n. 2073, de 28 de setembro de 2004. Instituí a Política Nacional de Atenção à Saúde Auditiva [Internet]. Brasília; 2004 [citado 2012 maio 12]. Disponível em: $<$ http://dtr2001.saude.gov.br/sas/PORTARIAS/ Port2004/GM/GM-2073.htm>. Acesso em 09/05/2016.

5. Brasil. Diretrizes de atenção da triagem auditiva neonatal universal. Brasília, 2012. Disponível em: http://bvsms.saude. gov.br/bvs/publicacoes/diretrizes_atencao_triagem_auditiva neonatal.pdf. Acesso em 21 fev 2017.

6. Brasil. Portaria $\mathrm{n}^{\circ} 1278$, de 20 outubro de 1999. Disponível em: http://bvsms.saude.gov.br/bvs/saudelegis/gm/1999/ prt1278_20_10_1999.html. Acesso em 20 nov2017.

7. Joint Committee on Infant Hearing. Year 2007 Position Statement: principles and guidelines for early hearing detection and intervention programs. Pediatrics. 2007; 120(4): 898-921.

8. Tochetto TM, Gatto CI. Deficiência auditiva infantil: implicações e soluções. Rev CEFAC. 2007; 9(1): 110-5.

9. Lewis DR, Marone ASR, Mendes BCA, Cruz OLM, Nóbrega M. Comitê Multiprofissional em Saúde Auditiva COMUSA. Brazilian Journal of Otorhinolaringology,2010; 76: p.121-128.Disponível em < http://www.scielo.br/ scielo.php? script $=$ sci_nlinks\&ref $=000121 \&$ pid $=$ S 1413 $6538201400040000800011 \& \operatorname{lng}=\mathrm{pt}>$. Acesso 30/05/2016.

10. Brasil. Ministério da Saúde. Lei $\mathrm{n}^{\circ} 12.303$, de 02 de Agosto de 2010. Disponível em: < http://www.planalto.gov. br/ccivil_03/_Ato2007-2010/2010/Lei/L12303.htm>. Acesso em $09 / 0 \overline{6} / 20 \overline{16}$

11. Brasil, Casa Civil. "Lei no 8.080, de 19 de setembro de 1990: Dispõe sobre as condições para a promoção, proteção e recuperação da saúde, a organização e o funcionamento dos serviços correspondentes e dá outras providências." Diário Oficial da união 128.182 (1990)

12. Sigolo C, Lacerda CBF. Da suspeita à intervenção em surdez: caracterização deste processo na região de Campinas/ SP. Jornal da Sociedade Brasileira de Fonoaudiologia. 2011; 23(1): 32-7. Disponível em < http://www.scielo.br/pdf/jsbf/ v23n1/v23n1a09>. Acesso em 01/06/2016.

13. Vieira GI. Política Nacional de Atenção à Saúde Auditiva: o caso do município de São Paulo. [Dissertação de Mestrado]. São Paulo: Faculdade de Saúde Pública da Universidade de São Paulo, 2013.

14. Brasil. Ministério da Saúde. Portaria n. 2073, de 28 de setembro de 2004. Instituí a Política Nacional de Atenção à Saúde Auditiva [Internet]. Brasília; 2004 [citado 2012 maio 12]. Disponível em: $<$ http://dtr2001.saude.gov.br/sas/PORTARIAS/ Port2004/GM/GM-2073.htm>. Acesso em 09/05/2016. 
15. Silva LSG, Gonçalves CGO, Soares VNM. Política Nacional de Atenção à Saúde Auditiva: um estudo avaliativo a partir da cobertura de serviços e procedimentos diagnósticos. CoDAS 2014; 26(3): 241-7. Disponível em: http://www.scielo. br/pdf/codas/v26n3/pt_2317-1782-codas-26-03-00241.pdf. Acesso em 13/12/17.

16. Schochat E. Avaliação eletrofisiológica da audição. In: Ferreira LP. Tratado de fonoaudiologia. São Paulo: Roca; 2004. P.656-68.

17. Bevilacqua, MC et al. A avaliação de serviços em Audiologia: concepções e perspectivas. Revista Brasileira de Fonoaudiologia. 2009;14(3):421-6. Disponível em <http://www.scielo.br/ scielo.php?pid $=$ S1516-80342009000300021\&script $=$ sci abstract\&tlng $=\mathrm{pt}>$. Acesso em 30/05/2016.

18. Viana ALD, Ibañez N, Elias PEM, Lima LD, Albuquerque M, Iozzi FL. Novas perspectivas para a regionalização da saúde. São Paulo Perspec 2008; 22(1): 92-106.

19. Canale A, Favero E, Lacilla M, Recchia E, Schindler A, Reggero N, Albera R. Age at diagnosis of deaf babies: a retrospective analys highlighting the advantage of newborn hearing screening. Int J Pediatr Otorhinolaryngol 2006; 70: $1283-1289$

20. Amado BCT, Almeida EOC, Berni PS. Prevalência de indicadores de risco para surdez em neonatos em uma maternidade paulista. Rev CEFAC 11. 2009; suppl 1: 18-23.

21. Pereira MCCS, Ribeiro LL, Sena EP. Influência do tempo de vida nos resultados da Triagem Auditiva Neonatal. Revista de Ciências Médicas e Biológicas 2015; 13.3: 313-318.

22. Ozcebe E, Sevinc S, Belgin E. The ages of suspicion, identification, amplification and intervention in children with hearing loss. Int J Pediatr Otorhinolaryngol 2005; 69(8): 1081-1087

23. Al-Abduljawad, Khayria A, Zakzouk SM. The prevalence of sensorineural hearing loss among Saudi children. International Congress Series. 2003; 1240. Elsevier
24. Rodrigues GRI, Loiola-Barreiro CM, Pereira T, Pomilio MCA. A triagem auditiva neonatal antecipa o diagnóstico e a intervenção em crianças com perda auditiva? Audiol Commun Res 2015; 20(3): 246-54

25. Béria JU, Raymann BCW, Gigante LP, Figueiredo ACL, Jotz G, Roithman R, et al. Hearing impairment and socioeconomic factors: a population-based survey of an urban locality in southern Brazil. Rev Panam Salud Publica. 2007; 21(6): 381-7.

26. Andrade CL, Fernandes L, Ramos HE, Mendes, CMC, Alves, C. Programa Nacional de Atenção à Saúde Auditiva: avanços e entraves da saúde auditiva no Brasil. Revista de Ciências Médicas e Biológicas 2014; 12 (4), 404-410. Disponível em: < http://www.portalseer.ufba.br/index.php/ cmbio/article/viewFile/9181/6749> Acesso em 13/06/2016.

27. Botelho FA et al. Prevalência de alterações auditivas em crianças de risco. Braz j otorhinolaryngol (Impr.) 2010; 76.6: 739-744.

28. Onoda RM, Azevedo FA, Santos AMN Neonatal Hearing Screening: failures, hearing loss and risk indicators. Braz J Otorhinolaryngol 2011; 77(6): 775-783

29. Pinto M, Melina, et al. Idade no diagnóstico e no início da intervenção de crianças deficientes auditivas em um serviço público de saúde auditiva brasileiro. Arquivos Internacionais de Otorrinolaringologia 2012; 16.1: 44-49.

30. Pereira A, Santana PK, Martins AS, Vieira MR, Azevedo MF. Programa de triagem auditiva neonatal: associação entre perda auditiva e fatores de risco. Pró-Fono Revista de Atualização Científica. 2007; 19(2): 267-278. Disponível em: < http://www.scielo.br/scielo.php?script=sci arttext\&pid $=$ S0104-56872007000300005 $>$. Acesso em 01/06/2016.

31. Barboza ACS et al. Correlation between hearing loss and risk indicators in neonatal hearing screening reference service. Audioly - communication Research 2013; 18(4): 285-292.

32. Olusanya BO, Newton VE. Global burden of childhood hearing impairment and disease control priorities for developing countries. Lancet 2007; 369(9569): 1314-7. 